\title{
La culture humaniste à l'École. Remarques sur la notion de « culture humaniste » et sur la formation au sein des ESPE dans le processus d'accompagnement des enseignant-e-s
}

\section{Salim Mokaddem}

\section{OpenEdition}

\section{Journals}

Édition électronique

URL : http://journals.openedition.org/trema/3339

DOI : $10.4000 /$ trema.3339

ISSN : 2107-0997

Éditeur

Faculté d'Éducation de l'université de Montpellier

Édition imprimée

Date de publication : 1 mai 2015

Pagination : $101-111$

ISSN : 1167-315X

Référence électronique

Salim Mokaddem, «La culture humaniste à l'École. Remarques sur la notion de « culture humaniste » et sur la formation au sein des ESPE dans le processus d'accompagnement des enseignant-e-s », Tréma [En ligne], 43 | 2015, mis en ligne le 25 juin 2015, consulté le 19 avril 2019. URL : http:// journals.openedition.org/trema/3339 ; DOI : 10.4000/trema.3339

Ce document a été généré automatiquement le 19 avril 2019

Trema 


\title{
La culture humaniste à l'École. Remarques sur la notion de «culture humaniste » et sur la formation au sein des ESPE dans le processus d'accompagnement des enseignant-e-s
}

\author{
Salim Mokaddem
}

«Ce qui est bien connu, justement parce qu'il est bien connu, est souvent méconnu ».Hegel

\section{Position du problème}

Nous pouvons nous demander pourquoi, après les programmes de 2005, 2008, 2013, l'importance de l'enseignement de la culture humaniste à l'École est plus que ré-affirmée, et, sollicitée activement par le Ministère de l'Éducation nationale ; en effet, les disciplines enseignées à l'École sont, par ailleurs, de façon directe ou transversale, porteuses de valeurs humanistes, comme le rappellent les récentes déclarations de Madame la Ministre Najat Vallaud-Belkacem, concernant les onze mesures d'urgence ${ }^{1}$ prises le 22 janvier 2015, pour prévenir, entre autres, un décrochage citoyen à l'École.

Quel est donc le rôle que peut jouer cette "culture humaniste ", cinquième compétence du socle commun de connaissances et de compétences ${ }^{2}$, dans la réussite scolaire ? Qu'en attend au juste le Ministère par sa mise en place dès la rentrée 2015-2016? Quelle formation faut-il mettre en place pour les professeur-e-s chargé-e-s de son enseignement ? De quelle «culture humaniste " s'agit-il, en fait, de faire vivre, de faire connaître et de savoir, à l'École ? Et, dans le même mouvement, quelle formation précise, épistémologiquement parlant, sera donnée aux formateur-e-s en charge de ces acquisitions spécifiques? 
Nous verrons alors que les objectifs louables, en principe, consistant dans l'introduction des valeurs de culture humaniste dans le système éducatif, ne s'accompagnent pas vraiment d'une visibilité de la définition pédagogique et philosophique de «la culture humaniste ». De même, sa déclinaison pédagogique pour et à l'École, demeure encore à préciser dans les modalités d'applications curriculaires. Est-il certain, d'abord, qu'existe une ou « la » culture humaniste, en soi ? Il nous faudra donc tenter, en un premier temps, de la définir, ou, à tout le moins, de comprendre ce que le Ministère en entend, pour plusieurs raisons, que nous analyserons en nous appuyant sur les compréhensions des «connaissances, capacités, attitudes (sic)» qu'est sensée développer ou enseigner l'introduction de «la » culture humaniste à l'École. Il est en effet légitime de s'interroger sur le et la portée de l'expression en elle-même (la culture humaniste): est-elle nominaliste (indicative d'un champ heuristique à définir singulièrement), réaliste (s'agitil d'un humanisme éclectique ou artificiellement reconstruit pour répondre à des besoins d'apprentissages spécifiques ?), ou bien, fait-elle référence à une périodisation singulière d'une histoire particulière (Renaissance française, italienne, positivisme du XIXe siècle, humanisme laïque d'une philosophie du progrès?), ou encore, sur un plan plus épistémologique, connote-t-elle une philosophie des savoirs et des connaissances (une vision hégélienne ou kantienne du progrès des savoirs dans l'Histoire du Monde ?) qui donnerait un contour actuel à un humanisme qui serait propre à notre présent ${ }^{3}$, qu'il resterait, par ailleurs, à définir? Autre hypothèse : cette expression, dans sa forme idéaliste, indique-t-elle une politique de la culture propre au champ éducatif? Relève-telle d'un projet, à proprement parler, idéologique, comme nous le pensons, qui viserait à instaurer une conception morale et civique des valeurs culturelles, pour les élèves et leurs enseignants, afin de déterminer une normalisation biopolitique des affects et des être-aumonde (le vivre-ensemble ${ }^{4}$ ) dans un monde complexe où les assignations sont, de plus en plus, incertaines à reconnaître et à affirmer, pour les élites et les gouvernances centralisées? Ne serait-ce pas alors le retour de l'instruction qui serait subrepticement introduit, au sens le plus normatif, à l'École, dans un cadre moralisateur et civique, pour éviter de s'interroger sur les rapports complexes entre savoirs scolaires et émancipation éthique des sujets soumis aux processus de normalisations du moment?

La polysémie de l'expression: "culture humaniste », et, tout aussi bien, son laconisme moral, méritent donc une herméneutique de son contenu, à tout le moins, une interrogation réflexive sur ses visées de sens. Nous ne ferons pas ici l'exposé du programme ou des séquences du curricula de « la culture humaniste à l'École ». Des sites spécialisés, tel Éduscol, des ouvrages officiels, s'en chargent déjà. Les Bulletins Officiels, les Instructions officielles, les sites académiques dédiés à la diffusion des outils pédagogiques et des banques de données à destination des enseignants, fourmillent de fiches d'exposition et d'outils appropriés permettant de savoir, en termes académiques, quel est le programme de cet humanisme scolaire, cycle par cycle, niveau par niveau, et, de prendre connaissance des attendus visés par sa mise en séquences pédagogiques au sein des classes afin de procéder aux évaluations à renseigner sur le fameux livret de compétences.

La question que nous voulons ici poser est de savoir quel est au vrai l'humanisme qui est mobilisé pour enseigner les valeurs recherchées par le législateur, à savoir, « la formation du jugement, du goût et de la sensibilité », et qui permettra "d'acquérir des repères en histoire (événements fondateurs), géographie (paysages et territoires, populations, etc.), en littérature et en arts (les grandes œuvres) $)^{5} »$. 
Il nous faudra alors réfléchir sur les compétences et les objectifs de ce curriculum, plus ou moins référencé, afin d'en déterminer autant les contours que les ambitions affichées, et, ainsi, ne pas en rester à une déclaration de principe, quelque peu formelle ou idéaliste, généreuse, mais, justement, qui se contente de points de vue généraux et régulateurs, au sens le plus kantien ${ }^{6}$ du terme, jusques et y compris dans les fameux documents d'accompagnement des enseignants, visant plus à se substituer à une démarche réflexive, par le fait qu'ils exposent davantage des modèles ou des paradigmes inspirateurs de séances que des problématisations ou des ensembles de thèmes à problématiser. Et, enfin, il est plus qu'urgent de nous demander si ce projet est cohérent, réalisable, enseignable, dans l'état actuel des programmes, et, surtout, au vu de la formation réelle des enseignants en France, dans les ESPE.

La culture humaniste, selon le législateur, a pour ambition de former le jugement, le goût et la sensibilité des élèves à l'École. Ce triple objectif, intellectuel, sensible, esthétique, correspond à une vision très platonicienne de l'éducation?

Il s'avère, dans sa formulation, essentialiste et prescriptif, autant que paradigmatique, supposant par sa formulation même, l'existence d'une anthropologie du juger, du sentir, de l'émotion; l'expression en elle-même indique qu'il y a une théorie des facultés cognitives, sensitives, réfléchissantes, relevant d'un kantisme affiché. L'élève, comme l'être humain, serait doué de tels dispositifs - et de dispositions affectives, émotives, cognitives - que l'École aurait pour mission de développer de façon presque spiritualiste. Le goût, le jugement, la sensibilité, n'auraient aucune assise dans le vécu social, familial, collectif, ou dans la praxis des élèves; ils seraient des éléments transcendantaux à faire croître selon une image relevant de la théorie génétique et critique d'un humanisme philosophique du sujet forclos sur lui-même, systématiquement enserré dans un rapport en soi à soi qui définirait le lien ontologique d'un sujet à sa vérité, indépendamment de l'épistémè qui le produit, de l'histoire qui le détermine, du langage qui conditionne ses pensées et l'ordre de ses désirs.

\section{1. Ce que dit le texte d'Éduscol : la culture humaniste ou le projet d'ouvrir l'esprit}

Le texte de référence pour le premier degré sur l'enseignement de la culture humaniste à l'École se trouve sur le portail national des professionnels du Ministère de l'Éducation nationale ${ }^{8}$, à destination des enseignants, nommé : Éduscol, site accessible par le lien suivant $^{9}$ : http://eduscol.education.fr/cid46267/competence.html.

Plutôt généraliste, ce programme indique un certain nombre de thématiques générales, sans définir toutefois les contenus spécifiques des œuvres et des pratiques dans les disciplines invoquées. Principalement, la culture humaniste est définie ainsi dans ce texte de cadrage :

«La culture humaniste contribue à la formation du jugement, du goût et de la sensibilité. Elle repose principalement sur la littérature, l'histoire, la géographie, l'éducation civique, les arts plastiques, l'éducation musicale ou encore l'histoire des $\operatorname{arts}^{10}$ \%.

D’emblée, et, en préambule, il est explicitement exprimé que «la » culture humaniste ${ }^{11}$ est littéraire, ou, à tout le moins, qu'elle ne prend pas en compte les sciences et les techniques (contrairement aux projets des encyclopédistes, ou des humanistes cartésiens, ou, même encore, à ceux du Quattrocento, de Da Vinci, qui ne séparaient certainement 
pas les sciences, les arts, les techniques et les beaux-arts $)^{12}$, mais qu'elle relève des sciences humaines et des beaux arts.

La valorisation des projets éducatifs et d'actions culturelles à l'École semble tenir lieu de placebo éducatif; la culture, l'art, les sciences humaines (et non pas les techniques, les sciences et les technologies) sont mobilisés pour lutter contre l'échec scolaire. Le focus mis sur ces plans «culturels» est ambitieux et participe d'une volonté sans doute généreuse, de réduire considérablement et durablement, les échecs scolaires et les décrochages divers qui grèvent les résultats du système éducatif français. Il a pour but affiché, en effet, de faire en sorte d'éviter que les logiques académiques, en cours dans les curricula et les établissements, ne surdéterminent davantage les pratiques sociales cultures élitaires et élitistes, en favorisant les privilèges symboliques, intellectuels, des héritiers ${ }^{13}$ par le jeu social-scolaire facilitant les connivences et les privilèges des enfants de " bonnes familles ", reconnus comme «bons élèves » par les évaluations sommatives et certificatives scolaires. Participant de pratiques insidieusement sélectives et d'exclusions logiquement structurées par le système de la production scolaire de la reproduction des inégalités sociales, les pratiques (et les théories) de l'École favorisent de manière inique les bénéficiaires, directs et indirects, de la lutte des places au sein même de l'École ${ }^{14}$, au nom même de l'«égalité des chances", qu'on ne confondra pas avec l'équité de répartitions des capitaux sociaux divers (réels et symboliques).

L'idée de cette Refondation de l'École, par la culture et les arts, résiderait dans la conception générale et généreuse que "la" culture, distribuée selon les axes du socle commun, permettrait aux élèves de s'ouvrir à l'altérité, à soi, au monde comme aux valeurs de la République, par une sorte d'éveil des consciences assez analogue à celui promu par les philosophes des Lumières du XVIIIe siècle européen. Cette idée ingénue, déjà dénoncée par Platon dans le Banquet, suppose alors que la mise en confrontation, plus ou moins médiée selon des protocoles peu précisés dans les programmes, avec le savoir, de la culture et des beaux arts, produirait des réveils ou des éveils réflexifs. Ainsi, la proximité ou le voisinage physique, la mise en contact, par une sorte d'opération mystérieuse (le fameux «bain » culturel), permettrait aux élèves d'« ouvrir » leur esprit par une confrontation révélatrice de leur esprit enfin sorti des ténèbres par les volontés conjuguées des pédagogies actives et des lieux consacrés, laïquement s'entend, par les institutions officielles chargées d'adouber, sinon de valider et de reconnaître les projets, les cadres, les lieux, les acteur-e-s, les œuvres les mieux profilés pour cette mission d'ouverture des esprits à la culture. Il y aurait donc, selon leur caractère de ferment de révélation culturelle, des lieux, des endroits, des personnes de références, ou, reconnues comme telles, par l'École qui reproduirait à l'extérieur de son cercle d'action traditionnelle et nouveau, une géographie de la culture sur le territoire redéfinissant ipso facto de nouvelles frontières, et de nouveaux champs, aux actions culturelles et citoyennes de l'École. Cette métaphore de l'ouverture de l'esprit au monde réclame un commentaire car on peut légitimement s'interroger sur la pertinence de la définition académique de la culture, en tant que telle, dans son processus d'" ouverture » aux autres cultures $\mathrm{du}$ monde ${ }^{15}$. Les partenariats entre les ministères ne feront pas oublier que l'éducation nationale n'est pas l'éducation culturelle (et nous ne parlons pas uniquement de celle diffusée et soutenue par le Ministère de la Culture), et, encore moins, que la formation du jugement nécessite d'autres formes d'enseignements que celles relevant de la logique industrielle de la production de normes abstraites, nécessairement relatives à des intérêts ne relevant pas uniquement de désintérêts de toutes sortes. L'humanisme 
scolaire - expression à prendre de manière critique - ne prend pas en compte, dans son fonctionnement immanent, les besoins effectifs des enfants et de leurs familles ${ }^{16}$, leurs histoires singulières, leurs langues premières, leur culture au sens strict (historique, et, indistinctement, généalogique). L'École et ses pratiques d'enseignement expriment de facto des méthodes, des logiques, des finalités, qui relèvent plus de la sélection par les évaluations, de la normalisation disciplinaire que de la formation éthique et philosophique du jugement, à proprement parler. L'École est d'autant plus exclusive qu'elle pense ne pas l'être.

Pourtant, il semble que ces parcours d'actions culturelles, ces apprentissages de culture humaniste à l'École, peuvent être confrontés à deux apories : celle concernant la culture disponible sur le territoire où s'exercent les projets culturels interinstitutionnels, d'une part, et, d'autre part, celle relevant de la culture professionnelle des enseignant-e-s. Les risques encourus sont donc de deux ordres : faire dépendre l'action culturelle de l'offre locale et ainsi entériner les inégalités d'offres sur les territoires, et, par ailleurs, laisser s'installer l'idée qu'une culture officielle d'état et d'État, décide de ce qui est culture et de ce qui ne l'est pas, par l'institutionnalisation de partenaires, d'associations, de lieux patrimoniaux ou mémoriels, d'acteurs reconnus ou non, de l'action culturelle locale, régionale, nationale. Il est patent que seules les cultures hétérogènes, les éruditions sauvages, les pratiques concrètes des praticien-ne-s et des professionnel-l-es de l'éducation pourront alors minorer ces effets négatifs contre-productifs de la surdétermination institutionnelle du culturel par l'École et ainsi tenter de faire en sorte que les reproductions ne soient pas survalidées par l'École en dehors de l'École, empêchant alors des contre-cultures ou des para-cultures de se manifester dans les marges restantes. Il est donc important que l'innovation et le travail dans des lieux reconnus, pensés et réfléchis comme emblématiques de multiplicateurs de démocratie acculturante, par des experts des formations professionnelles, soient à produire ou à reconnaître dans leur spécificité et leur idiosyncrasie. L'importance de ces choix significatifs de personnes et de lieux - et des modalités effectives des choix - est plus que nécessaire pour éviter l'uniformisation d'une culture officielle de l'entre-soi et un repli identitaire, presque communautaire ou de réseau, sur le terreau et le terroir culturel local ou de proximité, souvent en situation de rente réelle et symbolique en matière de capitaux culturels. Cette accumulation incestueuse de biens symboliques et réelspeut, de fait, reproduire les inégalités réelles des répartitions inégalitaires des dispositifs et des moyens de production et de diffusion des cultures scolaires, sociales, culturelles, déjà valorisées par l'École, contre les cultures dites de «consommation» ou de "divertissement», au détriment des culture in progress, des savoirs populaires, non savants au sens de l'École ${ }^{17}$.

\section{2. Le réveil de la raison engendre les monstres de l'humanisme triomphateur}

Que vise au juste cet enseignement de «la culture humaniste» (au sens objectivé que nous pointons dans cette étude)? Lisons la conclusion de présentation du socle commun 
de connaissances, de compétences et de culture, disponible en ligne sur le site Éduscol, pour bien en comprendre les enjeux :

«Attitudes

La culture humaniste que dispense l'école donne aux élèves des références communes. Elle donne aussi à chacun l'envie d'avoir une vie culturelle personnelle :

- par la lecture, par la fréquentation des musées, par les spectacles (cinéma, théâtre, concerts et autres spectacles culturels);

- par la pratique d'une activité culturelle, artistique ou physique. Elle a pour but de cultiver une attitude de curiosité :

- pour les productions artistiques, patrimoniales et contemporaines, françaises et étrangères ;

- pour les autres pays du monde (histoire, civilisation, actualité). Elle développe la conscience que les expériences humaines ont quelque chose d'universel. ${ }^{18}$ »

Que peut vouloir signifier l'expression : "l'envie d'avoir une vie culturelle personnelle » pour un élève ? L'envie provient-elle d'un désir subit lié à une motivation intrinsèque, à l'occasion de la fréquentation des œuvres de « la culture humaniste » ou est-ce un effet pavlovien d'imitation des auteurs étudiés ou approchés à l'École? Nous pouvons à juste titre, nous interroger sur la façon concrète dont va se réaliser cette conversion ou cette « envie » de culture :

- est-ce à dire que les élèves n'en auraient aucune si l'École ne les sollicitait en ce sens ? Seraient-ils donc abouliques et apathiques, en matière de "vie culturelle personnelle »?

- auraient-ils une (ou plusieurs) vie culturelle non personnelle (on peut ici supposer que le législateur veut prévenir ou contrevenir l'influence délétère de la famille, de la rue, du quartier, des médias, et, pire encore, de la culture populaire non scolaire) qui mettrait en danger leur socialisation scolaire et leur scolarité?

- qu'est-ce qu'une « vie culturelle personnelle » sinon un héritage de classe, de milieu, ou un habitus, à défaut d'être un don ou une grâce dont les élèves seraient bénéficiaires en dehors de l'École ? À moins que l'on suppose chez les élèves une capacité ou compétence spécifique pour naturellement se destiner vers les savoirs, les connaissances, les arts, les pratiques culturelles dont on sait qu'elles exigent souvent un long temps d'apprentissage et une familiarité d'usage et de valeurs que l'École n'a pas le temps, justement, de mettre à disposition, dans tous les sens du terme, pour les élèves?

- comment, une fois suscitée cette " envie d'avoir » (et non pas de vivre, ou de sentir ou d'être pleinement mais d'avoir) « une vie culturelle personnelle », comment donc l'École s'y prendelle pour aider ce conatus à se réaliser concrètement? Quel accompagnement est alors mis en place pour transformer une velléité en action et en pratique concrète, socialement établie, durablement installée dans la vie de l'élève, et, non pas, seulement, scolairement évaluée, et de ce fait, valable et valide uniquement dans l'enceinte et le cursus scolaire?

Il semble que ce lexique psychosocial, comportementaliste, sinon behavioriste, et ces imprécisions d'expressions idéalistes, cachent assez mal le renvoi à l'en-dehors de l'École, à un ailleurs de la classe, convoqué alors, implicitement, pour réguler, remédier, prendre en charge, soutenir, appuyer, les actions culturelles amorcées institutionnellement. Les élèves sont renvoyés à leur réalité de classe sociale en dehors de la classe scolaire. Ce qui signifie que les élèves sont, en dernière instance, renvoyés à leurs potentialités culturelles familiales, à des cultures propres, ou privées, au risque que les inégalités de parcours des familles ne soient, de ce fait, transmissibles à leurs enfants car l'accès économique, social, technique aux activités culturelles privatives extrascolaires relèvent justement de cultures codées privatives et d'héritages culturels des cellules familiales dans la société civile $^{19}$, en sus de leur coût souvent prohibitif pour les familles à qui l'on destine pourtant 
cette "culture humaniste » pour les ouvrir justement au monde qui ne les reçoit plus depuis longtemps pour des raisons relevant de secteurs non scolaires (logiques économiques, matérielles, géographiques, qui souvent se surdéterminent les unes avec les autres).

D'autre part, que signifie que la culture humaniste «développe la conscience que les expériences humaines ont quelque chose d'universel». Veut-on dire par là que les travaux de Lévi-Strauss, des historiens de l'École des Annales, des anthropologies culturelles, des philosophes de la différence ou de la complexité, ne sont rien et qu'il faut donc revenir à une vision anhistorique, transhistorique, suprahistorique de la "conscience» et ainsi ne pas prendre en compte les déterminations sociales, linguistiques, phénoménologiques, culturelles, matérielles, dans la formation de "la" conscience ? Ce « quelque chose d'universel » dont il est question n'est-ce pas le retour à la vieille question de la nature de l'homme dont le XXe siècle a eu beau jeu de nous montrer l'archéologie religieuse, le fondement politique basé sur une biopolitique des âmes et du contrôle de soi ? Ne s'agit-il pas là dans ce programme de balayer la question de l'histoire, de la détermination rigoureuse des effets des pensées et des actions des hommes sur eux-mêmes et leur entourage, dans une prise en compte à la fois de la matérialité discursive et éthique de leur être-au-monde ? L'universel est une construction historique et la prise en compte de l'historicité des volontés de savoir et de faire relève, aujourd'hui, d'une démarche philosophique en dialogue constant et rigoureuse, avec les interrogations et les connaissances dont nous sommes à la fois les effets et les fins dernières et premières. Notre modernité, fût-elle post ou transhumaniste, ne se pose plus les questions de l'Autre sous la forme d'une transcendance à forclore dans un sujet qui en détiendrait la vérité totale et définitive.

L'universel de «la » culture humaniste a-t-il en soi une valeur morale qui transcenderait les cultures particulières pour atteindre la " personne » de l'enfant, plus durable que celle de l'élève ? Y a-t-il des vérités philosophiques éternelles que la culture humaniste de l'École peut révéler et éveiller en l'enfant de telle sorte que son envie ne soit pas annihilée par le jeu de la mise à l'écart social ? Comment les enseignant-e-s s'y prendront-ils pour lutter contre le poids des déterminations sociales et les pesanteurs sociétales qui obèrent les parcours scolaires des élèves issus de milieux défavorisés? Quelle est la culture humaniste que doivent apprendre les formateurs pour éviter que cette culture ne soit le reflet d'un monde qui exclue, par l'usage de « la » culture, les cultures de ce type d'élèves que l'École maintient à distance pour reproduire les conditions de la reproduction de leur exclusion structurelle? Quelle anthropologie culturelle peut dépasser les particularismes et les situations historiques singulières, déterminées par des réalités spécifiques, pour porter cette "culture humaniste", universelle, transcendante aux singularités et aux réalités des vécus des enfants ? Est-il par ailleurs certain que les professeur-e-s des Écoles, formé-e-s sur le modèle des classes préparatoires du fait des nouvelles maquettes des mastères - fussent-ils professionnels - des ESPE (cours magistraux, TD portant sur ces cours, évaluations en série, mémoire formel coupé des stages pratiques) auront le temps de prendre le temps de leur formation à «la culture humaniste» sur leur temps de préparations aux concours et aux diplômes leur permettant d'entrer dans le métier par la voie du recrutement par ces évaluations? Les ESPE ne peuvent, dans l'état actuel des maquettes universitaires, et, au vu de la part menue faite aux stages en responsabilité dans les Écoles, répondre à ces questions de formation ; c'est, conscient de cela, pour ces raisons précisément, que des injonctions rémanentes à la formation des formateurs et des 
acteurs sont invoquées dans les circulaires et autres missives performatives autant que phatiques du Ministère. Comment former les cadres pédagogiques sans s'interroger à la fois sur les contenus des cultures de l'humanisme en France, en Europe, dans le Monde? Est-il possible ne pas prendre en compte les acquis des sciences humaines sur les impasses des humanismes au XIXe et au XXe siècle?

N'oublions pas que l'humanisme européen mena au colonialisme planétaire, et, que des totalitarismes très différents, en théorie et en pratique, se réclamèrent d'un surhomme comme d'un homme nouveau, au nom d'un humanisme neuf, universel, total, qui a exclu de l'humanité réelle, plus qu'il ne les a intégrés, les enfants, les femmes et les hommes concrets, réellement humains, des cultures et des civilisations du Monde dont ces humanismes européens faisaient la conquête guerrière tout en proclamant qu'elles les menaient vers le progrès, vers les Lumières de la culture, par le glaive sanglant de l'éducation culturelle.

L'humanisme peut-il jamais se décliner autrement qu'en particularité, culturelle et civilisationnelle, ethnocentrée?

\section{Conclusive aporie}

Ce sont là des questions que les enseignant-e-s en formation et les étudiant-e-s des ESPE devront se poser, de manière rigoureuse et fine, pour éviter que « la » culture à l'École ne devienne un mot d'ordre creux et purement abstrait, visant à écarter, une fois de plus, les plus démunis, ou les damnés de l'École, qui sont encore, majoritairement, les exclus de la réussite scolaire. L'idée d'humanisme ne doit pas écraser l'humanité réelle qu'elle est censée représenter. Un réalisme culturel instruit peut, peut-être, éclairer de manière délicate, les oublis d'un nominalisme moralisateur portant encore, par devers soi, comme une ombre tragique, une métaphysique, essentialiste et civilisatrice, aux accents guerriers dont l'écho a du mal à se transformer en acculturation pacifique et progressiste, aujourd'hui encore, comme le révèle une actualité tragique. Car, malgré les promesses d'harmonie universelle que chantent les injonctions à plus de République, ici et là, en une époque qui argue trop souvent de l'insécurité sociale et historique pour limiter trop rapidement les libertés politiques, définir un humanisme pratique du construire ensemble passe par l'interrogation philosophiquement fondée sur les logiques de territorialisation et d'exclusion de l'humanisme universel niant les anonymes et silencieuses, non moins que cruelles, partitions par lesquelles il s'autoconstitue.

N'oublions pas que le ministère de l'» Instruction publique », celui du fameux Ministre des hussards noirs de la République, Jules Ferry, fut aussi celui des colonies, banlieues de l'histoire universelle ${ }^{20}$. Il se peut qu'à oublier les conditions historiques de l'apparition effective de l'École actuelle, nous soyons soudainement pris par un souci d'humaniser son réel et, partant celui des élèves, au point de réveiller l'ordre profond qui la structure depuis longtemps déjà, et, ainsi, de faire réapparaitre, sous une forme à la fois inédite et trop connue, la face la plus sombre et coercitive de son archéologie militaire. 


\section{BIBLIOGRAPHIE}

Bergson, H., (2012), Le bon sens ou l'esprit français, éd. Arthème Fayard, « Mille et Une nuits ", $\mathrm{n}^{\circ}$ 610.

D’Alembert, J., (Le Rond), (2000), Discours préliminaire de l'Encyclopédie, éd. Vrin, Paris.

Foucault, M., (1966), Les mots et les choses, éd. Gallimard, Paris ; L'archéologie du savoir, ibid. (1968) ; La société punitive. Cours au Collège de France, 1972-1973, « Hautes études », éd. GallimardSeuil-EHESS, Paris,

Glissant, E., (2010), Entretien pour Télérama du 8 juillet, « La pensée unique frappe partout où elle soupçonne de la diversité », consultable avec le lien : http://www.telerama.fr/idees/edouardglissant-et-son-tout-monde,58073.php

Heidegger, M., (1946), Lettre sur l'humanisme, trad. R. Munier, éd. Montaigne, Paris, 1957.

Kant, L., Traité de pédagogie, éd. Vrin, trad., Paris.

Legendre, P., La Fabrique de l'homme occidental, Arthème Fayard, Mille et Une nuit.

Merleau-Ponty, M., (1947), Humanisme et terreur, éd. Gallimard, Paris.

Mokaddem, S., (2014), in Dialektiké, nº 1, Brasil.

Mokaddem, S.,(2014), Ce que nous apprend le hip hop, in Collectif, éd. Centre Chorégraphique National de La Rochelle.

Platon, République, (1803), éd. et trad., Robin, Gallimard, Euvres complètes, « La Pléiade », Paris.

Sartre, J.-P., (1946), L'existentialisme est un humanisme, Paris, éd. Nagel.

Sayad, A., (2014), L'École et les enfants de l'immigration. Essais critiques, Seuil, Paris.

Wilde, O., (2013), L'âme de l'homme sous le socialisme, éd. Arthème Fayard, « Mille et Une nuits », n 613, trad. J. Cantel.

\section{Sitographie sélective}

http://www.education.gouv.fr/cid2770/le-socle-commun-de-connaissances-et-decompetences.html\&xtmc=culturehumanistealecole\&tnp=1\&xtcr=3

http://cache.media.education.gouv.fr/file/01-

janvier/49/4/2015_DP_mobilisation_Ecole_complet_385494.pdf

\section{NOTES}

1. Mesures qu'on peut lire, par exemple, sur le site Éduscol : http://www.education.gouv.fr/ cid85644/onze-mesures-pour-une-grande-mobilisation-de-lecole-pour-les-valeurs-de-larepublique.html

2. On se reportera, avec profit, au Décret $n^{\circ} 2006-830 \mathrm{du} 11$ juillet 2006 comme texte de référence. 
3. Présent qui, rappelons-le, ne se soucie plus de l'humanisme comme courant de pensée et porte plus sur le post-humanisme initié par la pensée de Nietzsche ou le transhumanisme influencé par le progrès des sciences (biologie et cybernétique pour faire court) et des techniques. On relira avec intérêt les travaux de P. Legendre, P. Sloterdijk, autant que ceux de G. Agamben, pour comprendre les enjeux politiques, éthiques et philosophiques, de ces questions épineuses des «fins» de l'homme. La destination de ces «fins» demeure plus qu'aporétique si l'être de l'humanité est de ne pas avoir d'essence autre que celle de sa négativité historique.

4. Nous préférons plutôt un "construire ensemble " à un vivre-ensemble, assez peu actif et quelque peu physique : car, en effet, on peut très bien vivre-ensemble de façon contiguë sans solliciter, pour soi et pour autrui, un horizon de projet(s) commun(s).

5. Cf., sur le site du Ministère de l'Éducation nationale, les définitions du socle commun de compétences et de connaissances, et les formulations concernant la compétence $5:$ http:// www.education.gouv.fr/cid2770/le-socle-commun-de-connaissances-et-de-

competences.html\&xtmc=culturehumanistealecole\&xtnp=1\&xtcr=3

6. Le principe régulateur d'une Idée, au sens de Kant, sert à guider la raison, en tant que concept pur de la raison, sans intuition sensible permettant de la réaliser ou d'en faire l'objet d'une connaissance objective dans le champ transcendantal ou empirique. L'idée régulatrice relève donc d'un idéal de la raison pure dans le champ moral (pratique) et spéculatif (métaphysique), mais, elle ne peut jamais se réaliser dans une politique de la raison pure. Kant fait de l'éducation une idée vraie absolument, même si elle est impossible, en fait, comme le rappelle le Traité de pédagogie de 1803.

7. On se rappellera que pour Platon, dans la République, la paidéia a pour but de mettre l'âme en ordre hiérarchique (Nous, thumos, épithumia ou raison, volonté, sensibilité) selon un arrangement à la fois ontologique et éthique correspondant à une classification axiologique des vertus et des puissances de dévoilement de l'être et du Bien. Le but de l'éducation, selon Platon, étant de faire une Cité belle et juste, à partir de l'Idée du Bien qui commande l'organisation rationnelle des facultés de l'âme des citoyens, eux-mêmes reconnaissant la sagesse des roisphilosophes à laquelle se soumettent gardiens et artisans, selon un ordre indistinctement éthique, métaphysique et politique.

8. http://eduscol.education.fr/cid46267/competence.html

9. Dans le cadre de ce court article, nous nous référons uniquement à l'indicatif de ce programme.

10. Ibid.

11. Il serait intéressant de préciser quel est, au juste, cet " humanisme » dont se réclame ce curriculum, car, en effet, cette essentialisation de la culture humaniste suppose une réalité invariante, transhistorique, ne dépendant ni d'aucun lieu, ni d'aucun temps, pour parler comme Fénelon caractérisant la morale de l'étude historique selon lui. Cependant, les historiens (Burckhardt, Vernant, Dumézil, Bloch, Febvre, Duby, entre autres) et les philosophes (Nietzsche, Hegel, Heidegger, Foucault) ont démontré qu'il y a plusieurs humanismes, et que l'humanisme particulier de la Renaissance italienne n'a rien à voir avec, par exemple, celui du positivisme du XIXe siècle, celui de Comte ou encore moins avec celui de l'existentialisme sartrien (qui n'est pas l'humanisme de Merleau-Ponty, celui de Marx, ou encore, celui de Dewey), pour faire bref. Des penseurs du siècle passé se sont même opposés, épistémologiquement, à l'humanisme classique et contemporain, au prétexte qu'il portait avec lui des transcendances naturalisées non réfléchies (Bataille, Althusser, Canguilhem). Nous reviendrons in fine sur cette naturalisation culturelle, ou d'essentialisation, processus de réification gommant les idiosyncrasies historiques, et, par cette opération dialectique, indiquant la pré-éminence, de manière abstraite, de l'existence d'un transhistorique universel et indéterminé, socle commun de toutes les expériences de culture, visant à rejeter les singularités, les spécificités, les concrets historiques dans les poubelles de l'histoire ou les insignifiances anecdotiques de la prose du monde, pour reprendre les termes par 
lesquels Hegel, au nom du progrès de la Raison dans l'Histoire, rejetait rapidement ce qui ne faisait pas système avec la Logique du Concept. Justement, l'humanisme du siècle précédent se voulait un retour à l'Homme plus qu'à un être intemporellement situable ou à une Transcendance abstraite, sans odeur, ni couleur, sans lieu, ni langues vivantes, fût-elle appeler « Humanité ». Ce qui obligera les philosophes du concept à être anti-humanistes en théorie pour protéger l'humanisme pratique ou réel. Ce fut tout le sens de la querelle entre les tenants du structuralisme versus ceux du sujet, au sens classique et moderne du terme. On relira avec profit la conclusion de M. Foucault, in Les mots et les choses, Paris, Gallimard, 1966, et, L'Archéologie du savoir, Paris, Gallimard, 1968, pour mettre en situation ces débats philosophiques qui tracent encore les contours de notre actualité.

12. Il suffit de lire le Préambule du Discours préliminaire de l'Encyclopédie pour constater, chez Diderot et d'Alembert, les intrications et imbrications nettes entre sciences, philosophie et émancipation du genre humain. C'est même, au vrai, le projet explicite des Lumières.

13. Pierre Bourdieu et Jean-Claude Passeron, Les héritiers. Les étudiants et la culture, éd. de Minuit, Paris, 1964.

14. Rappelons, pour mémoire, que selon l'enquête Pisa publiée par l'OCDE, en décembre 2013, la France a été indexée négativement comme étant le pays le plus inégalitaire dans son système scolaire du fait de l'importance que joue les profils socio-économiques des parents dans la réussite scolaire de leurs enfants.

15. Le sociologue Abdelmalek Sayad, dans L'École et les enfants de l'immigration, éd. du Seuil, «La couleur des idées », Paris, 2014, montrait déjà, dans un article de 1977, repris dans l'opus cité, la non reconnaissance par l'École des cultures des enfants issus de l'immigration de leur parent.

16. Michel Foucault, dans Surveiller et punir, éd. Gallimard, Paris, 1975, avait déjà pointé les archéologies disciplinaires des systèmes éducatifs occidentaux.

17. Cf. Salim Mokaddem, "Ce que nous apprend le hip hop », La Rochelle, 2014, revue du CCN.

18. Éduscol, ibid.

19. C'est pour cela que les TAP (temps d'activité périscolaires) ont été institués à et dans l'École, au demeurant, afin de pallier les inégalités extrascolaires influençant les parcours académiques des enfants selon leurs origines sociales, culturelles, économiques. Il est temps de faire le bilan de ces TAP sur les réussites scolaires des élèves concernés.

20. Le ministre de la Refondation, Vincent Peillon - qui n'a pas voulu porter jusqu'à son terme la réforme initiée par lui, préférant siéger comme député européen, à Bruxelles - dans une geste très proche de l'intention kantienne, mais, fort peu proche de la praxis réelle de l'éthique de l'agir, ne cache pas le caractère moral de l'éducation selon lui. Philosophe de formation, ayant œuvré dans les ex-Iufm, Vincent Peillon, est aussi féru de philosophie bergsonienne et se réclame assez des choix spiritualistes et laïques de la loi de 1905, de Combes et de Buisson, plus que de ceux de 1936 et de l'esprit de la constitution de 1958. Pour comprendre le sens de ce pédagogisme spiritualiste français, nous recommandons la lecture de H. Bergson, Le bon sens ou l'esprit français, éd. Mille et Une nuits, Paris, 2012, qui reprend des textes intégraux de Bergson (1895, 1923, 1934) sur l'enseignement, les études classiques, et, la réforme de l'enseignement secondaire. Les similitudes spiritualistes et conservatrices, à l'intérieur des courants pédagogiques du socialisme philosophique en France, mériteraient, en soi, un travail de synthèse particulier, pour comprendre la rémanence d'un discours atemporel et tourné plutôt vers un passé, souvent idéalisé, chez les pédagogues républicains, depuis Condorcet jusqu'à aujourd'hui. Pour le lien entre éducation à l'universel et colonialisme, le poète des caraïbes, Édouard Glissant, fait cette remarque critique à propos de l'universel, et des diversités qu'il exclut : « Les cultures occidentales ont inventé la notion d'universel pour imposer en fait leurs propres "valeurs " comme valables pour tous et nous imposer la notion même de valeur. Cette catégorie d'universel est une tromperie complète. Les gens qui la prônent refusent la diversité du monde. La France, 
par exemple, continue de croire (du moins ses dirigeants) que l'assimilation pure et simple des Antilles à la France est la seule voie envisageable ou légitime pour ces pays, et le seul mode de relation possible ». (Entretien publié le 8 juillet 2010 pour Télérama).

\section{RÉSUMÉS}

Que veut dire aujourd'hui pour nous enseigner et apprendre «l'humanisme» et «les humanités " dans les écoles françaises? Au vrai, les programmes balaient un très grand spectre de connaissances historiques, et, aussi des champs imprécis de savoirs plus ou moins déterminés. Pourtant, ils sont les produits d'une épistémé bien spécifique. La " mort de dieu », thématisée par le postmodernisme, nous contraint de réfléchir aux conséquences logiques de ce nihilisme sur « la mort de l'Homme ». Pouvons-nous encore enseigner un humanisme sans prendre en compte le déclin épistémologique du concept d'humanité dans les sciences sociales contemporaines? Nous devons nous interroger sur les fondements autant éthiques qu'archéologiques d'une renaissance de l'humanisme pour penser ce que sont vraiment les valeurs de l'humanisme culturel et de l'humanisme politique si nous ne voulons pas nous contenter d'affirmations idéologiques. Un regard philosophique s'avère nécessaire pour déterminer précisément ce qu'est un humanisme pour et dans l'École car l'être humain demeure encore à ce jour un topos aporétique.

What does it mean for us today: Teaching and Learning «Humanism» and «Humanities» in french schools? In fact, the curricula schedules a very large of historical knowledges, and an accurateless field of undetermined sciences. Social sciences are historical constructions. So, they are underlined with specific epistémé. Remembering what postmodernism use to call the Death of God, we could reflect that the Death of Man is a logical consequence of this nihilism. Could we again teach humanities without considering the decay of humanism in the true shapes of postmodern sciences? We have to undertake an ethical and archeological reappraisal of the humanities to think what does really mean the values of cultural humanism and a political humanism if we don't want to schedule ideological assents about them. We need a philosophical point of view to precise what is humanism for and in schools because human beeing does remain still now an aporetical topos.

\section{INDEX}

Mots-clés : enseigner un humanisme, humanités, valeurs éducatives

Keywords : humanities, teaching humanities, values of cultural humanism

\section{AUTEUR}

\section{SALIM MOKADDEM}

Faculté d'Éducation - Université de Montpellier, ESPE-LR, Chercheur associé au Laboratoire LIRDEF 